\title{
Les espaces de travail connectés : une perspective nouvelle pour la modélisation dans le secondaire?
}

\author{
Jean-baptiste LAGRANGE \\ Laboratoire de Didactique André Revuz. Université de Paris. \\ jb.lagrange@casyopee.eu
}

\section{Cite this article}

Lagrange, JB. Les espaces de travail connectés : une perspective nouvelle pour la modélisation dans le secondaire?. Can. J. Sci. Math. Techn. Educ. (2021). https://doi.org/10.1007/s42330-020-00130-6

\begin{abstract}
Our interest is in the development of modeling activities that link knowledge in various mathematical fields and other scientific disciplines. Looking at mathematics education research we highlight the benefits and limitations of "problem-solving” and of approaches that involve translation between the real world and mathematics. Science education seldom takes mathematics into account. Its contribution is the conception of an “empirical referent”. Using epistemological studies, we highlight examples of modeling situations supported by a plurality of models and an organization of work that allows students to engage with them. To do this we adopt the theoretical framework of connected workingspaces. A case study shows how students create linkages between the workspaces and the benefits of the connections on their understanding of concepts at stake.
\end{abstract}

\section{Keywords}

Modelling, math education research, working spaces, connections.

\section{Résumé}

Nous nous intéressons à la conception d'activités de modélisation pour la mise en relation de connaissances dans divers champs des mathématiques et d'autres disciplines scientifiques. En didactique des mathématiques, nous soulignons les apports, mais aussi les limites des approches "problem solving" et "translation entre réalité et mathématiques". La didactique des sciences prend quant à elle peu en compte les mathématiques. Son apport est dans la conception d’un "référent empirique". A partir d’études épistémologiques, nous privilégions des situations de modélisation s’appuyant sur une pluralité de modèles et une organisation du 
travail des élèves qui leur permette de confronter ces modèles. Nous adoptons pour cela le cadre théorique des espaces de travail connectés. Un exemple de situation montre comment les élèves créent des connexions entre espaces de travail et les apports de ces connexions à leur compréhension des concepts en jeu.

\section{Mots clés}

Modélisation, didactique des mathématiques, didactique des sciences, espaces de travail, connexions.

\section{Motivation}

Cet article s’intéresse aux activités de modélisation par les élèves du secondaire, à la conception de ces activités, à leur mise en œuvre et à leur évaluation. Il fait suite à l'expérimentation d'activités par un groupe où travaillent ensemble des enseignants et des chercheurs $^{1}$ dans le contexte du lycée Français, motivées par les limites ressenties dans les approches courantes en éducation mathématique. Ces activités se caractérisent par une approche de la modélisation basée sur la confrontation de plusieurs modèles d'une même réalité à partir d’une question générale. L’objectif est pour les élèves la mise en relation de savoirs concernant divers champs des mathématiques, ainsi que d'autres disciplines scientifiques. Cette mise en relation, et si possible en cohérence, de différents savoirs nous paraît un objectif important en fin de secondaire où les élèves disposent d'une variété de savoirs scientifiques mais ont peu l'occasion de les faire fonctionner en synergie et d'en concevoir l'utilité en dehors du champ disciplinaire où ils sont enseignés. Il s’agit aussi de faire prendre conscience aux élèves du rôle que jouent les modèles dans ces disciplines et dans la vie sociale. Dans cet article nous prenons comme exemple la modélisation d'un câble porteur dans un pont suspendu ${ }^{2}$. Une annexe précise les éléments de référence : la question est "quelle est la forme d'un câble porteur ?"33 et elle peut être vue sous l'angle de quatre modèles se situant chacun dans un champ scientifique spécifique de l'enseignement secondaire : sciences physiques, géométrie, algorithmique et fonctions mathématiques (Fig. 1). Nous cherchons à approfondir les éléments théoriques et pratiques permettant la conception et l'évaluation d'une situation d'apprentissage et à ouvrir ainsi des perspectives nouvelles pour les activités de modélisation au secondaire.

\footnotetext{
${ }^{1}$ Le site http://casyopee.eu présente le groupe et donne des exemples d'activités.

${ }^{2}$ Cet exemple a été présenté dans différents colloques. Voir par exemple (Lagrange \& Le Feuvre 2019).

${ }^{3}$ Un pont suspendu comporte généralement deux câbles porteurs symétriques pas rapport au plan médian longitudinal. C’est pourquoi nous écrivons "un câble porteur" plutôt que "le câble porteur".
} 


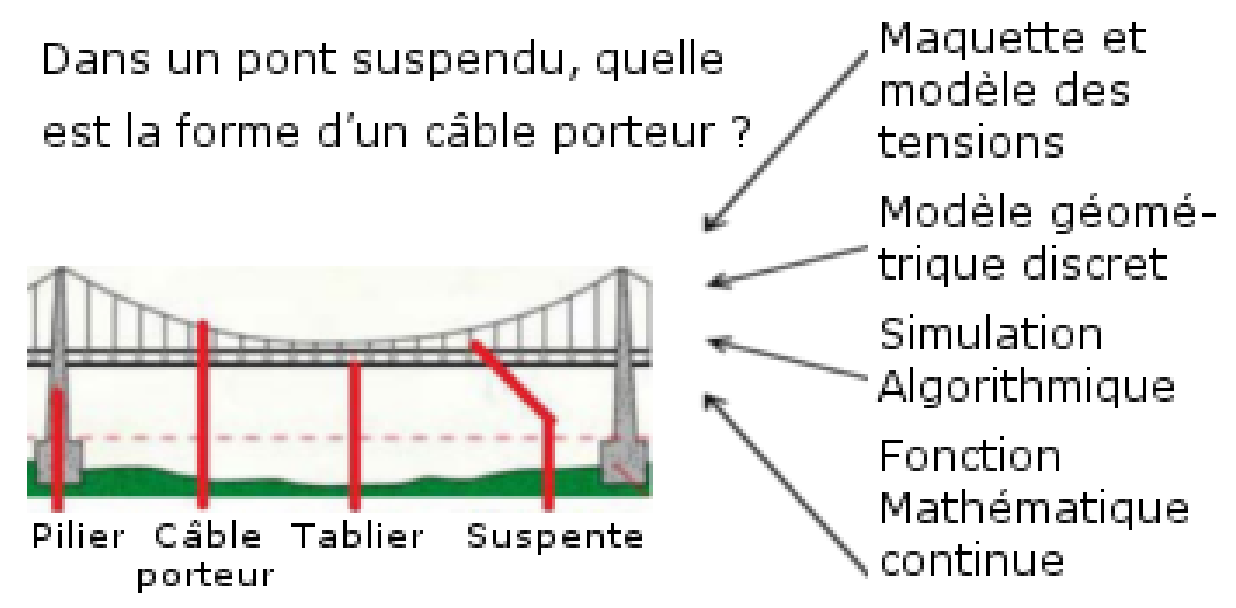

Fig. 1 : Une question et quatre modèles.

\section{La modélisation dans l'enseignement des mathématiques et des sciences}

Dans cette section, nous précisons en quoi les approches courantes en éducation mathématique nous ont semblé avoir des limites. Nous repérons deux grandes tendances dans l’enseignement des mathématiques, l’inscription dans la "résolution de problèmes" et la "translation entre le réel et les mathématiques". Par ailleurs la mise en place de situations de modélisation avec les objectifs précisés plus haut conduit à collaborer avec des enseignants et chercheurs d'autres didactiques (les sciences physiques dans l'exemple) et il convient donc d’étudier comment la modélisation est vue dans ces didactiques.

\section{La résolution de problèmes}

De façon à se démarquer des "word problems", de nombreux auteurs caractérisent les activités de modélisation comme la résolution de problèmes "authentiques". Kaiser et Schwarz (2009) se donnent ainsi pour objectif de conduire les élèves à faire l'expérience de la puissance de la modélisation mathématique pour la compréhension et la résolution de tels problèmes. Ils se situent donc dans une perspective "problem solving" qui est souvent adoptée dans la littérature sur la modélisation. Ils mènent un travail empirique auprès d'élèves du secondaire au cours d'une "semaine de modélisation" hors temps scolaire. L’observation porte sur la résolution d'un problème de répartition d’arroseurs dans un jardin. On constate un travail très riche des élèves pouvant conduire à la comparaison de solutions exprimées à l'aide de formules mathématiques. Il y a donc bien "mathématisation". En revanche, on ne voit pas apparaître explicitement un ou des modèles. Certes les formules mathématiques obtenues pour la surface arrosée pourraient être considérées comme des modèles de cette surface, mais elles ne sont pas analysés comme telles dans l'article et ne font pas l'objet d'une réflexion de la 
part des élèves. L’évaluation par questionnaire montre que ceux-ci ont bien perçu la mise en relation des mathématiques avec la "vie réelle". En revanche, en ce qui concerne leur travail, ils mettent surtout l'accent sur la résolution de problèmes : ils soulignent l’intérêt du travail en équipe et la possibilité d’approcher un problème par plusieurs voies, et aussi le caractère chronophage des activités proposées et le rapport peu évident avec les contenus enseignés, aspects souvent évoqués à propos du "problem solving".

Ainsi, beaucoup d’activités proposées dans la littérature sur la modélisation nous semblent mettre finalement l'accent sur la solution à un problème contextualisé plutôt que sur des modèles. Certes, les élèves développent certains processus de modélisation, mais le modèle reste sous-jacent et est oublié quand la solution est trouvée. Ainsi dans Blum et Ferri (2009), l'énoncé porte sur un phare de hauteur donnée et demande de trouver une valeur de la distance de visibilité valable spécifiquement pour cette hauteur. Les auteurs repèrent chez les élèves des "pas" cohérents avec le "cycle de modélisation" qui est leur cadre théorique, mais ces pas conduisent à la solution plutôt qu'à un modèle.

\section{Translation et cycle de modélisation}

Les recherches sur la modélisation dans l'enseignement des mathématiques adoptent aussi très souvent des approches du type "translation" entre réalité et mathématiques. Elles prennent comme point de départ des problèmes ou des situations qui font sens dans l'expérience quotidienne des élèves. Ces situations étant nécessairement plus complexes que celles des "word problems", elles donnent lieu à une étape de simplification et de structuration. Cette étape suppose que les élèves mobilisent leur compréhension des éléments contextuels du problèmes en vue de repérer et d’organiser ceux qui sont pertinents et d'éliminer les autres. Blum et Ferri (ibid.) appellent "modèle réel" le résultat obtenu. Il est attendu ensuite des élèves qu’ils "mathématisent" la situation ainsi simplifiée et structurée, obtenant ce que Blum et Ferri, appellent un "modèle mathématique". En cohérence avec la perspective "problem solving" mentionnée plus haut, aucun élément mathématique n’est indiqué dans la tâche et donc, implicitement, cette étape consiste pour les élèves à faire un choix parmi des structures ou procédures mathématiques déjà rencontrées, et à instancier ces éléments pour la situation. Dans de nombreuses recherches, cette étape est conçue de façon unidirectionnelle et se ramène à une "mise en équation". Dans d'autres, comme dans l'exemple du phare cité plus haut, on peut observer des mouvements inverses du "modèle mathématique "vers le "modèle réel". Décrites le plus souvent dans le cadre d’un "cycle de modélisation" ces étapes de constitution de modèles "réel" puis "mathématique", sont suivies d’une étape de résolution. 
Les deux dernières étapes du cycle reviennent vers la situation en traduisant le résultat mathématique dans les formes propres au domaine (repérage de la grandeur en jeu avec ses unités), puis son interprétation en fonction du problème posé. Il y a "translation" au cours de la phase de "mathématisation" et au cours de ces deux dernières étapes. Littéralement, "translation" signifie "déplacement sans modification de structure" et donc ces approches ne considèrent pas des processus qui relèveraient de l'adaptation, de la transposition, de la conversion ou de la mise en correspondance ${ }^{4}$.

\section{L’intégration du raisonnement dans la réalité avec le raisonnement mathématique}

Finalement, le cycle de modélisation reprend, en précisant les étapes et les transitions, le schéma de résolution de problème "extra-mathématique" sur deux niveaux, le problème et la solution étant exprimés au niveau "extra-mathématique" et la résolution étant opérée au niveau mathématique. Il y a un apport certain qui permet notamment d’interpréter les trajectoires de résolution des élèves. Néanmoins, le "réel" et le "mathématique" restent deux niveaux peu imbriqués ce qui, comme nous l'avons vu plus haut, met l'accent sur la démarche d’accès à une solution plutôt que sur le modèle. Tout en se situant dans la perspective du cycle de modélisation et de la résolution de problèmes, Czocher (2018) montre qu’il peut exister une relation plus riche entre contexte du problème et mathématiques. Elle tire parti d’un travail empirique sur la résolution de problèmes par des élèves ingénieurs. Les connaissances tant mathématiques que relatives au contexte de ces élèves leur permettent des choix variés d'entrée dans le problème et on observe de fréquents retours en arrière sur les choix relatifs au "modèle réel" aussi bien dans l’étape de "mathématisation" que dans les étapes ultérieures. Écartant la modélisation comme simple "translation", Czocher considère que la modélisation fait intervenir une "intégration" du raisonnement dans la réalité avec le raisonnement mathématique. Elle note que le cycle de modélisation "peut ne pas donner une juste idée de l'activité de l'apprenant au cours de la modélisation et met probablement un accent trop important sur une division entre un monde réel et un monde mathématique" (p. 153, notre traduction) .

Le "raisonnement dans la réalité" mis en évidence par Czocher ne repose pas sur le simple bon sens, il fait intervenir des connaissances scientifiques non formelles, qui permettent aux

\footnotetext{
${ }^{4}$ Selon Blum et Ferri (2009, p. 1) "Mathematical modelling (is) the process of translating between the real world and mathematics in both directions". "Translation" peut se comprendre comme "traduction" (entre deux langues, celle de la réalité et celle des mathématiques)", ce qui est une conception souvent admise, par exemple dans le curriculum français (MEN/DGESCO-IGEN 2013, p.1), aussi bien que "déplacement" entre domaines. Nous gardons "translation" car, dans les recherches considérées, les aspects langagiers ne sont pas dominants.
} 
élèves ingénieurs de formuler des hypothèses, puis de les contrôler en raisonnant à l'aide de l'appareillage mathématique introduit lors de la mathématisation, et aussi de contrôler ce raisonnement en évaluant sa capacité à tirer parti des hypothèses. Ces contrôles croisés donnent davantage corps à l'idée de modèle que dans les exemples précédents, puisque ce n’est pas la solution au problème ni la démarche qui sont en jeu ici, mais les choix structurels qui les sous-tendent. Ces contrôles donnent lieu aussi à des interactions fortes entre connaissances scientifiques, certes non formalisées, et connaissances mathématiques. C’est ce type de raisonnement qui est visé dans l'exemple de la modélisation du câble porteur d'un pont suspendu : on souhaite que les élèves fassent référence aux entités physiques considérées dans le premier modèle quand ils raisonnent sur les autres modèles plus directement mathématiques, en associant notamment une partie infinitésimale de la courbe de la fonction recherchée à une barre élémentaire constitutive du câble "discret", et en reconnaissant le paramètre $H$ intervenant dans les autres modèles comme la composante horizontale de la tension dans le premier modèle.

\section{Dans l'enseignement des sciences expérimentales, un travail sur le "référent empirique"}

Puisque, à côté des mathématiques, des champs scientifiques présents comme disciplines d'enseignement tels que les sciences physiques ou les sciences de la terre peuvent intervenir dans la modélisation, il nous faut interroger les approches dans leur enseignement. Nous analysons un article représentatif de tendances assez générales en didactique des sciences. Sanchez (2008), à la suite d'une large revue de travaux mentionnant la modélisation dans les sciences expérimentales et leur enseignement, propose un ensemble de tâches conduisant l'élève à travailler sur les caractéristiques et propriétés d'un modèle : identification des différents éléments constitutifs du modèle et des relations hiérarchiques ou causales qui lient ces éléments, et mise en place de simulations par lesquelles les élèves peuvent identifier les conséquences de modifications de paramètres du modèle et en évaluer la cohérence interne. Même si les mathématiques ne sont pas explicitement mentionnées, les relations entre éléments du modèle et les paramètres des simulations devraient donner une place aux mathématiques.

Cette impression disparaît quand l'auteur présente un travail empirique en enseignement de la géologie. Ce travail porte sur l'appréhension par des élèves de lycée d'un modèle de lithosphère en trois couches. La tâche des élèves est de mener une étude de terrain pour repérer des formations conformes au modèle et d'autres qui s'en éloignent. On voit les élèves s’approprier des éléments du modèle pour diriger leur étude et en rendre compte, mais aussi 
des difficultés parasites liées à la collecte de données par des non-spécialistes. La tâche ne réalise qu'une petite partie de l'ensemble dont l'auteur traçait les contours, et qui aurait pu conduire à prendre en compte la dynamique de formation des couches en mobilisant des concepts physiques et des méthodes mathématiques. En dépit de cette limitation l'auteur attribue à cette tâche de collecte et de confrontation à un modèle une place centrale dans l'enseignement des sciences au secondaire. En fait, sa préoccupation est celle des rapports entre un "référent empirique" ${ }^{5}$ constitué d'objets et de phénomènes tels qu’ils sont perçus par les élèves et de connaissances qu’ils mobilisent spontanément, et un "référent scientifique" constitué d'éléments théoriques. Cette préoccupation domine aussi dans la revue de travaux en didactique des sciences que l'auteur présente en introduction. Le modèle sert à mettre en relation ces deux "référents", ce qui contribue à ce que, chez les élèves, ils progressent ensemble. En effet, la problématique en sciences expérimentales n’est pas de substituer chez les sujets un "référent scientifique" au "référent empirique", mais plutôt de rendre ce dernier efficace en l'articulant à des éléments théoriques.

Pour un didacticien des mathématiques, cette situation paraît limitée par comparaison à ce que sont aujourd'hui les sciences de la terre et l'implication des mathématiques ${ }^{6}$. Le choix de Sanchez est représentatif d'une tendance dans l'enseignement des sciences expérimentales à limiter la technicité des modèles à cause des difficultés mathématiques rencontrées par les élèves, tendance que regrettent Blanquet et Picholle (2015). Il serait néanmoins dommage de ne pas tirer parti d’une approche de ce type, car ce qui y est désigné comme " référent empirique" est ce qui est mobilisé par les élèves pour le "raisonnement dans la réalité". Pour les activités de modélisation que nous visons, cela implique un travail spécifique dans une ou des disciplines impliquées dans la modélisation. Dans l'exemple présenté en annexe, un travail sera nécessaire en préalable au travail sur les modèles, amenant les élèves à distinguer les ponts suspendus parmi d'autres ouvrages de franchissement et à approfondir l'idée de tension dans un câble.

\footnotetext{
${ }^{5}$ L’auteur emploie "registre empirique" et "registre scientifique" plutôt que "référent empirique" et "référent scientifique", tout en précisant que "registre empirique" est un raccourci pour "registre du référent empirique". Le terme "registre" est employé en didactiques des sciences expérimentales dans l’acception commune de catégorie de représentation sans la signification précise qu’il a pris en didactique des mathématiques sous l’influence de Duval (1995). Nous intéressant ici aux objets plus qu’à leurs représentations, nous substituons « référent » à « registre » ce qui permet d'éviter une discussion sur la notion de registre qui nous entraînerait trop loin.

${ }^{6}$ voir le site http://www.breves-de-maths.fr
} 
Terminons par une remarque : les articles analysés plus haut sur la modélisation dans l'enseignement des mathématiques et des sciences expérimentales ont une ambition principalement "cognitive". Face à un problème extra-mathématique, quelles vont être les démarches des élèves ? Que révèlent ces démarches sur leur rapport aux mathématiques et sur l'évolution de ces rapports ? Comment peut-on les faire évoluer ? Ces questions ne nous sont pas étrangères -nous visons la mise en relation de divers savoirs en mathématiques ainsi que d'autres disciplines scientifiques, mais dans cet article notre préoccupation est d'abord épistémologique. Comme le dit Sierpinska (1999)

"il y a décidément des moments où les chercheurs se préoccupent surtout des contenus mathématiques : ils essaient de définir ce qu’ils veulent enseigner, de le comprendre, de le mettre dans la perspective des objectifs généraux d'enseignement, de voir ses liens avec d'autres objets d'enseignement. Ils cherchent des problèmes qui donneraient du sens à ces contenus pour les élèves." (Sierpinska 1999, p. 154)

Ces préoccupations nous semblent peu prises en compte dans les recherches où le plus souvent la "modélisation" et particulièrement le rapport que les mathématiques (ou les sciences) entretiennent avec le contexte en jeu sont considérés comme "allant de soi". Sierpinska (ibid.) souligne aussi que les questions cognitives et épistémologiques sont "entremêlées" et même dans le cadre de cet article nous ne pourrons pas y échapper. Cependant nous nous limiterons à un postulat assez général sur les apprentissages dans le cadre des activités de modélisation. Ce postulat sera énoncé plus loin.

\section{La modélisation : quelle interaction entre le "réel" et les mathématiques?}

La section précédente a interrogé des tendances concernant la modélisation dans l'enseignement des mathématiques et des sciences expérimentales. Visant la conception d’activités pour la mise en relation de connaissances dans divers champs des mathématiques et d'autres disciplines scientifiques, nous soulignons les apports, mais aussi les limites, des approches "problem solving" et "translation". Il s’agit pour nous de concevoir une interaction fructueuse entre le "réel" et les mathématiques, ou plus précisément d’articuler "raisonnement dans la réalité" et "raisonnement mathématique". Le "raisonnement dans la réalité" doit être compris comme s'appuyant non sur le sens commun, mais sur des concepts scientifiques mobilisés dans l'action, et donc les activités doivent inclure un travail sur un "référent empirique" propre au domaine modélisé sur lequel la didactique des sciences met l’accent. Ce 
but étant précisé, il reste à rechercher la spécificité des mathématiques dans la modélisation. Sous quelles formes interviennent-elles ? Quelles sont leurs fonctions?

\section{Mathématisation et modélisation}

Roux (2011) signale la difficulté à définir de façon univoque la mathématisation, tant les domaines d'intervention des mathématiques et les formes que prennent ces interventions sont variés. Elle repère néanmoins (p. 6) des "formes" qui peuvent caractériser des mathématisations. Ce sont la quantification ("l’association de certaines propriétés à des quantités") et la formalisation ("les cas où l’application des mathématiques devient significative par la puissance de la forme"). La mathématisation d’un domaine peut alors être vue comme la systématisation de démarches de quantification et de formalisation, jusqu’à faire du raisonnement mathématique déductif le mode dominant.

Cependant, pour Roux (ibid. p. 17) il existe des interventions des mathématiques qui n’ont pas cette visée. Ces interventions différencient la modélisation de la mathématisation et se caractérisent i) par la localité : "alors que la mathématisation a une visée globale, la modélisation est toujours locale, et pour tout dire, fragmentaire : le modèle s'assume comme étant seulement une manière parmi d'autres de rendre compte non pas de la réalité, mais de certains de ses aspects seulement" ; ii) par l’opérativité : "alors que la mathématisation se présente comme une théorie ayant pour seule fin le savoir, la modélisation a une dimension opérative et interventionniste : un modèle est un dispositif qui permet d'agir sur ce qui est modélisé, que ce soit en le suppléant, en le contrôlant ou en le modifiant". La mathématisation comme théorie mathématique d'un domaine peut certes contribuer aux moyens d'action sur le domaine mais ce n’est pas sa visée première et il n’est pas toujours nécessaire ou possible d’y faire appel pour agir. ${ }^{7}$

Un corollaire de la caractérisation i) est la multiplicité des modèles pour une réalité donnée. Bouleau (2014) commence son ouvrage par l'exemple du trafic automobile et identifie une variété de modèles sur quatre niveaux, chacun tenant compte d'aspects de la complexité du trafic et de sa mesure. Il souligne (p. 19) qu’au premier niveau, les modèles simples "sont très faux à cause de leur simplicité mais sont aisés à ajuster car ils nécessitent relativement peu de mesures" et que à d'autres niveaux, des modèles s’approchent mieux des phénomènes, mais "ils se servent de (...) paramètres qu’on n’a pas les moyens de connaître". Il en déduit que "l’initiation à la modélisation et à sa critique est essentielle pour former les ingénieurs et les scientifiques qui auront à agir pour faire avancer les décisions collectives ..."

\footnotetext{
${ }^{7}$ Nous ne retenons pas un troisième critère, "le désengagement ontologique".
} 
L’exemple donné en annexe s’inscrit modestement dans cette perspective : parmi les quatre modèles proposés, aucun n’est plus "juste" que l’autre, mais chacun "explique" à sa façon la conception d'un pont suspendu. Varenne (2014, p.29) fait écho à la caractérisation ii) en soulignant la proximité entre modèle et simulation. Une simulation peut être un modèle "phénoménologique" rendant compte seulement des aspects visibles de la réalité modélisée, ou au contraire reposer sur une modélisation plus en profondeur. Dans ce cas, Varenne parle de modèle "à la seconde puissance". Parmi les modèles de l'exemple en annexe, deux s’apparentent à la simulation : dans le premier modèle, une maquette matérielle est obtenue en représentant le tablier comme un ensemble de masses disjointes et dans le modèle algorithmique, une simulation sur ordinateur est proposée. Nous écartons un modèle purement phénoménologique qui pourrait être obtenu en cherchant par régression une fonction dont la courbe "s’adapte" à une image réelle car nous visons la compréhension d'éléments fondamentaux de la réalité modélisée et non la seule solution d'un problème. Toujours concernant la caractérisation ii) selon Bouleau (ibid., p. 29) il ne faudrait pas que le caractère opératoire du modèle occulte une composante "interprétative" qui concerne le sujet engagé dans une modélisation et donc son contexte social. Par cette composante, la modélisation se distingue de la mathématisation où le raisonnement déductif met de côté les enjeux sociétaux. Dans l'exemple présenté en annexe, une discussion pourrait avoir lieu sur les modèles et leurs limites, mais elle serait sur un plan "technique" plus que "sociétal" portant par exemple sur les choix qu'un ingénieur serait amené à faire et l'éclairage que donne chaque modèle sur ces choix.

Nous devons donc distinguer les démarches de mathématisation (quantification, formalisation, raisonnement ...) qui peuvent intervenir dans la modélisation d'un domaine, de la mathématisation de ce domaine qui systématise ces démarches. La modélisation se caractérise par (1) la pluralité des modèles qui permet de rendre compte d’aspects locaux d’une réalité, (2) l’opérativité et donc une proximité avec la simulation, (3) une composante interprétative subjective et sociale.

\section{Les "sciencettes"}

Pour Roux et pour Varenne, l’objet d'étude est la modélisation dans des domaines où le contexte social est celui de la recherche, alors que Bouleau (ibid. p. 30) s’intéresse à des domaines comme le trafic automobile qu'il appelle "sciencettes"8, porteurs d'enjeux sociétaux

\footnotetext{
${ }^{8}$ Le terme "sciencette" pourrait paraître malheureux s’il impliquait une hiérarchie entre une ou des sciences "nobles" et des domaines scientifiques professionnels ou "appliqués" qui ne sont pas soumis aux mêmes critères. Les exemples tels que la résistance des matériaux, l'optique de Gauss, la thermodynamique des gaz parfaits et
} 
plus larges et non soumises aux contraintes de "falsifiabilité". Pour un domaine d'expérience donné, une "sciencette" permet d’aborder la réalité de façon scientifique et dans son contexte social à la différence des situations "quotidiennes" qui servent de base à un modèle "réel" résultant de simplifications qui ne mobilisent que le sens commun. Les sciencettes ont aussi l'avantage de mobiliser de façon coordonnée des notions dans divers champs des mathématiques et des sciences physiques, ce qui répond bien à notre objectif de mise en synergie de connaissances dans les domaines scientifiques, sans toutefois que les critères de rigueur soient nécessairement celles du raisonnement mathématique. Dans l'exemple donné en annexe, passer d'une modélisation discrète à la courbe d'une fonction dérivable, demande de considérer la limite de la tension en chaque point du câble quand le nombre de suspentes tend vers l'infini. Un raisonnement mathématiquement rigoureux est délicat ${ }^{9}$ et présente peu d’intérêt pour nos objectifs. Un meilleur choix est de viser un raisonnement de type "physicien" ou "ingénieur" en assimilant la courbe recherchée à la ligne brisée du modèle discret, les segments étant de longueur infinitésimale. Transposer une "sciencette" va ainsi dans le sens d’une "modélisation authentique", en s’inspirant des moyens intellectuels et matériels que se donnent les spécialistes du domaine plutôt que des seuls "problèmes".

\section{Le travail mathématique dans la modélisation}

Dans les recherches épistémologiques sur la modélisation vues ci-dessus, les mathématiques sont toujours présentes. Pour Bouleau (2014, p. 48), l’apport des mathématiques ne résulte pas de la translation d'une réalité déjà simplifiée à des mathématiques, mais du travail du mathématicien qu’il décrit comme "la recherche d’idées simples sans lesquelles les modèles seraient trop complexes pour être interprétables". Ainsi le mathématicien ne substitue pas un modèle mathématique à un modèle directement issu du domaine à modéliser ; il développe des mathématiques au sein de ces modèles pour les rendre plus intelligibles. Dans l'exemple présenté en annexe, établir des relations de récurrence pour les deux premiers modèles (statique et géométrique), construire une structure itérative pour le modèle algorithmique et calculer une fonction par intégration dans le quatrième modèle, sont des manières de rendre intelligibles l'évolution de la tension et son influence sur la forme du câble.

l'économie de la concurrence parfaite montrent bien que l'utilité sociale et le caractère rationnel de ces domaines sont indéniables et les distinguent de pseudosciences telles que l'astrologie. Nous conservons ce terme par fidélité à l'auteur, en remarquant qu'il peut dénoter une certaine autodérision puisque Nicolas Bouleau a une formation d’ingénieur des ponts et chaussées et d'architecte.

\footnotetext{
${ }^{9}$ Voir https://casyopee.math.univ-paris-diderot.fr/articles.php?lng=fr\&pg=206
} 
Bouleau consacre un chapitre entier aux "mathématiques, ressource conceptuelle et syntaxique." Il insiste sur le fait que, dans la recherche de la simplicité, le mathématicien n’est pas guidé par "des procédés déductifs sur la syntaxe" (ibid., p. 45), mais plutôt par l’intuition, en rapport avec le domaine à modéliser. Nous repérons ainsi deux dimensions du travail mathématique dans la modélisation : la dimension "sémiotique" qui concerne les systèmes de signes utilisés pour représenter la réalité et la dimension "discursive" qui concerne le raisonnement mathématique en lien avec le raisonnement dans la réalité. Nous développerons ces dimensions plus loin et y ajouterons une dimension "instrumentale" en nous inspirant du cadre des Espaces de Travail Mathématiques (ETM) de Kuzniak, Tanguay et Elia (2016).

\section{But et choix pour des situations de modélisation}

L’étude qui précède nous permet de préciser notre but. Les situations de modélisation pour le secondaire doivent pouvoir s’appuyer sur :

1. une pluralité de modèles pour mettre en jeu des connaissances scientifiques appartenant à des champs variés ;

a. des modèles proches du réel qui soient l'occasion pour les élèves de mobiliser des connaissances scientifiques dans l'action,

b. des modèles où les mathématiques interviennent pour faciliter l'interprétation, faisant intervenir des champs différents (géométrie et analyse...) et des méthodes différentes (calcul et simulation...) ;

2. une organisation du travail des élèves qui leur permette de confronter ces modèles et de faire fonctionner ces connaissances en interaction.

Nos choix sont au nombre de trois, et nous les présentons en montrant comment dans l'exemple de l'annexe, ils conduisent à une situation d'apprentissage, conçue pour des élèves de Terminale Scientifique (12 grade, 17 à 18 ans).

\section{Choix $n^{\circ} 1$. Partir d'objectifs dans un champ de connaissances et construire une situation que les concepts et méthodes de ce champ permettent d'éclairer.}

Dans notre exemple, il s'agit d'articuler le discret et le continu dans le calcul différentiel et plus précisément de concevoir l'approximation d'une courbe par une ligne brisée, et donc de considérer les notions de pente en un point d'une courbe et de nombre dérivé à la lumière de cette approximation. L'étude de la courbe formée par les câbles principaux d’un pont suspendu est bien adaptée en ce sens que le nombre fini de suspentes conduit à un modèle du câble en ligne brisée et que le grand nombre de suspentes permet de "passer à la limite" vers un modèle comme courbe d'une fonction continue dérivable. D’un point de vue physique la 
notion centrale est celle de tension dans un câble. Il s'agit d'une notion que les élèves ont rencontrée lors d'exercices de statique mais qui n’est pas familière.

\section{Choix $\mathbf{n}^{\circ}$ 2. Un cadre théorique permettant la mise en place a priori de la situation et l'interprétation a posteriori des observations.}

Il doit rendre compte des aspects fondamentaux de la modélisation dégagés dans les sections précédentes : multiplicité et confrontation des modèles, travail sur le "référent empirique", spécificité du travail mathématique dans chaque modèle, accent mis sur la modélisation plutôt que sur la solution à un problème. Nous adoptons pour cela le cadre des espaces de travail connectés proposé par Minh et Lagrange (2016) pour l'enseignement des fonctions. Ce cadre s’inspire de Kuzniak (2013) qui présente les espaces de travail géométriques comme un moyen d'éviter les malentendus dans l'enseignement de la géométrie. Le travail dans un espace donné se caractérise pour nous par trois dimensions ${ }^{10}$ : une dimension "sémiotique" où il s’appuie sur une ou des représentations, une dimension "instrumentale", avec l’usage d'artefacts et une dimension "discursive" qui met en jeu le raisonnement et s'appuie sur des théorèmes et autres règles d’inférence. Ainsi, en géométrie, pour une tâche donnée, on peut distinguer le travail dans un espace où les instruments jouent un grand rôle dans le raisonnement avec un système de signe réduit en s’appuyant sur des propriétés "naturelles", et le travail dans un autre espace où le raisonnement s’opère principalement par déduction avec le système de signes étendu du langage géométrique en s’appuyant sur un corpus précis de propriétés démontrées ou admises, les instruments jouant plutôt un rôle heuristique. L'analyse de Kuzniak montre que chacun des deux espaces a sa rationalité propre et que donc il ne s’agit pas de les opposer. Il s’agit plutôt de caractériser ces deux rationalités différentes de façon à pouvoir les articuler dans un projet didactique, par exemple lors de la transition d'une géométrie de l'enseignement primaire vers celle du secondaire. Le cadre des ETM est adopté dans de très nombreux travaux en didactique parmi lesquels certains prennent en compte l'articulation de plusieurs espaces de travail ${ }^{11}$; Minh et Lagrange (2016) ont initié la construction d'un cadre théorique rendant compte de cette articulation dans le domaine des fonctions.

\footnotetext{
${ }^{10}$ A la différence de Kuzniak (2013) et Kuzniak, Tanguay et Elia (2016) nous ne distinguons pas les plans épistémologique et cognitif, et donc nous ne convoquons pas la notion de genèse. Ce choix est cohérent avec notre préoccupation principalement épistémologique ; le qualificatif "dimension" a déjà été employé par des auteurs tels que Derouet \& Parzysz (2016) pour la même raison.

${ }^{11}$ Sans chercher l'exhaustivité, on peut citer Derouet et Parzysz (2016) articulant les probabilités avec l'analyse et Moutet (2019) sur la relativité vue du point de vue des sciences physiques et de celui des mathématiques.
} 
Dans cet article nous considérons que le travail sur un modèle s’inscrit dans un espace qui lui est propre avec une représentation sémiotique de ce qui est modélisé, des instruments pour agir sur cette représentation et un mode de raisonnement spécifique. Plusieurs modèles d'une même réalité, chacun inscrit dans un espace de travail, permettent de construire des "connexions" entre ces espaces de travail. Ce choix découle de l’analyse épistémologique de la section précédente : pluralité des modèles et place spécifique du travail mathématique. Il nous permet de préciser le postulat annoncé à la fin de la première section : par des "connexions" entre espaces de travail, les élèves progressent dans leurs connaissances scientifiques en les mettant en interaction, et comprennent les enjeux de la modélisation.

Dans l'exemple, à partir des quatre modèles détaillés en annexe, nous considérons quatre espaces de travail. Dans le premier espace, le câble est représenté comme un ensemble de "barres rigides" dans chacune desquelles s’exerce une tension. Cette représentation est concrétisée par une maquette, les tensions sont manipulées avec le formalisme de la physique, les instruments sont des dispositifs concrets de mesure -dynamomètres et rapporteur- et le raisonnement s’appuie sur la loi d'équilibre statique. Nous appelons cet espace de travail, l'espace des systèmes statiques, ou en raccourci l'espace de la statique. Le deuxième espace de travail porte sur une représentation géométrique discrète d'un câble porteur (une "ligne brisée"). La règle principale est la définition analytique d'un segment : les élèves peuvent calculer les coordonnées du point, connaissant les coordonnées de l'autre point, la pente et la différence entre les abscisses. Les instruments de tracé peuvent être utilisés. Nous appelons cet espace de travail l'espace géométrique. Dans le troisième espace de travail, la représentation du câble est une simulation informatique. Un algorithme calcule une série de points au moyen d'un traitement itératif. Ces points définissent une fonction par morceaux continue dont le graphique constitue la simulation du câble. Nous appelons cet espace de travail l'espace algorithmique. L'instrument est l'outil informatique sur lequel s'exécute l'algorithme de simulation. Enfin, dans le quatrième espace, la représentation du câble est une fonction mathématique. Elle est obtenue par intégration d’une fonction représentative de la pente du câble en chacun de ses points. C'est l'espace de travail des fonctions mathématiques avec leur symbolisme propre, les théorèmes de l'analyse et la possibilité d’utiliser des instruments de calcul. 


\section{Choix $\mathbf{n}^{\circ}$ 3. Des modalités de mise en œuvre découlant des choix précédents.}

Le groupe Casyopée utilise un dispositif, celui de "la classe en puzzle"12, qui a l'avantage de bien distinguer le travail dans différents espaces. Les modalités de travail sont en quatre phases : (1) les élèves s’approprient le thème en réactivant des connaissances antérieures ; (2) les élèves sont répartis en "groupes d'experts" ; chaque groupe approfondit un aspect spécifique du thème à partir d’un document et d'autres supports, et d'une tâche à réaliser ; (3) les groupes sont mixés et dans les nouveaux groupes, dits "groupes d'apprentissage", chaque expert développe son aspect du thème pour les autres, et le groupe met en cohérence ces aspects et élabore un compte rendu ; (4) une synthèse a lieu en classe entière. Dans une activité de modélisation nous choisissons de faire travailler les élèves non pas sur un "problème" au sens de résultat à trouver comme dans les approches "problem solving", mais sur une "question" susceptible de mettre l'accent sur un ou des modèles à construire ou à découvrir à la suite d'une recherche. La phase 1 est une entrée dans la question et vise à constituer chez les élèves le "référent empirique" introduit plus haut. Les aspects traités par chacun des groupes de la phase 2 correspondent à différents modèles et donc à différents espaces de travail. Nous attendons dans la phase 3 que les modèles soient confrontés et donc que des connexions entre espaces de travail soient construites. La synthèse de la phase 4 permet de faire collectivement le point sur les modèles et leurs connexions.

\section{La modélisation d'un câble porteur d'un pont suspendu}

\section{Organisation, tâches et analyse a priori}

Dans l'exemple, la question posée est, comme nous l'avons dit, celle de la "forme d'un câble porteur". La phase 1 dure une heure et vise d'abord à initier les élèves aux questions liées aux ponts. Ils sont invités à consulter un site web dédié pour repérer et esquisser quatre ponts de différents types, à regarder une vidéo illustrant l'idée de tension le long d'un câble horizontal et le fait que, même pour une tension importante dans ce câble, il n'est plus rectiligne dès qu'une force, même faible, est appliquée verticalement en un point. Ils doivent répondre à trois questions : (i) pourquoi dans un pont suspendu les câbles porteurs ne sont-ils pas rectilignes ? (ii) la forme d'un câble porteur est-elle déterminée par la longueur des suspentes ? (iii) A priori quelles fonctions peuvent modéliser un câble porteur ? On s'attend pour (i) et (ii) à ce que les élèves mentionnent l'effort exercé par le poids du tablier, mais aussi à des confusions concernant le rôle des suspentes, les élèves les supposant réglées pour

\footnotetext{
${ }^{12}$ inspiré de la "jigsaw classroom" https://fr.wikipedia.org/wiki/Jigsaw classroom.
} 
donner une certaine forme au câble à partir d'un tablier supposé indéformable. Pour (iii) il s’agit de préparer le questionnement des phases ultérieures.

Toujours dans cette première phase, les élèves doivent réaliser deux montages comme dans la Fig. 2, lire les tensions dans les dynamomètres et mesurer les angles, calculer les composantes horizontale et verticale des tensions et vérifier l'équilibre statique des forces. Pour le premier montage, les élèves devraient reconnaître un "triangle des forces" classique en statique. Le second montage est plus complexe : le segment central est horizontal par symétrie, par conséquent la composante verticale est nulle ; la loi d’équilibre statique donne pour les deux autres segments une composante horizontale égale à celle du segment central et une composante verticale égale au poids de chaque masse. Dans les deux cas la décomposition des forces en composantes horizontale et verticale pourrait poser problème aux élèves aussi bien pour l’observation que pour la vérification de la loi d’équilibre statique.

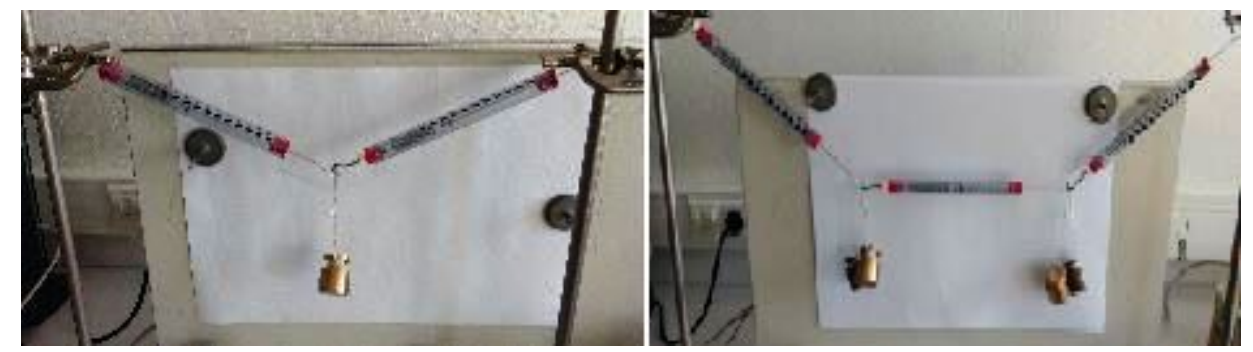

Fig. 2 : Les montages de la phase 1.

La phase 2 dure 50 min. Au début les données relatives au pont du Golden Gate sont présentées à toute la classe. Les élèves regardent aussi une maquette commentée par le professeur (Fig. 3) où le tablier est modélisé par un ensemble de masses raccordées aux suspentes. Les dynamomètres permettent de visualiser la tension dans chaque barre et un élève lit les tensions dans ces dynamomètres à toute la classe. Les élèves devraient ainsi prendre conscience d'une représentation adéquate du tablier comme suite de masses suspendues, et de ce que la tension varie en intensité le long du câble.

La tâche A concerne l'espace de travail de la statique et un modèle du câble comme ensemble de barres rigides : comme dans le travail de la première phase, les élèves doivent prendre en compte la suite des composantes verticale et horizontale des tensions dans les barres, reconnaître que la composante horizontale est constante et calculer une formule pour la suite des composantes verticales. La difficulté pour les élèves sera de raisonner en un point de suspension arbitraire (d'indice $i$ ) pour calculer les composantes des tensions au point suivant (d'indice $i+1$ ). Il est probable qu'ils ne pourront aller au-delà et trouver une formule explicite pour la suite des tensions. 


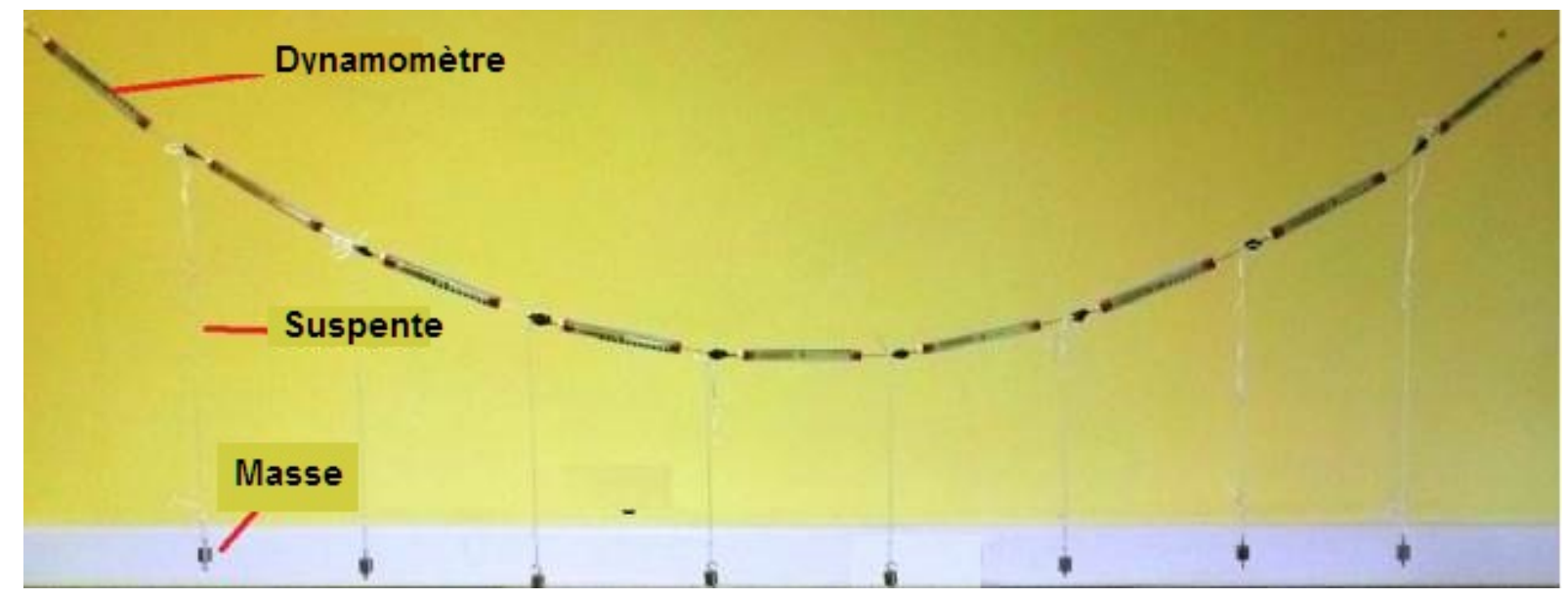

Fig. 3 : Une maquette d'un câble porteur.

La tâche B concerne l'espace de travail géométrique. Les données sont celles du pont du Golden Gate et on se place dans un repère centré au milieu du tablier. Il est indiqué aux élèves que le câble est modélisé comme une suite de segments $\left[M_{i} ; M_{i+1}\right]$ et une formule est donnée pour la pente de chaque segment en fonction de son indice $i$ et d'un paramètre $H$. Il est demandé d'exprimer la pente de chaque segment en fonction de l'abscisse de $M_{i}$ et de trouver une relation de récurrence pour les coordonnées des $M_{i}$. Les élèves ont les connaissances nécessaires en géométrie analytique, mais ici aussi il ne va pas leur être facile de raisonner sur des suites de points et de pentes, les calculs faisant de plus intervenir une constante $H$ indéterminée.

La tâche $\mathrm{C}$ se situe dans l'espace de travail algorithmique. Les élèves doivent entrer et exécuter l'algorithme de la Fig. 4 dans un environnement de programmation permettant une simulation dynamique du câble comme une suite de segments ${ }^{13}$. L'algorithme dépend de deux paramètres $n$ et $H$ que les élèves doivent faire varier et interpréter, le premier comme le nombre de points de suspension et le second comme une "tension" dont la modification influe sur la forme du câble. Au moment où nous avons expérimenté cette situation, l’algorithmique était encore peu abordée au lycée et nous avons choisi une tâche d’interprétation, plutôt que de conception d'algorithme. Cette tâche est facilitée par l’insertion de commentaires faisant le lien avec le pont du Golden Gate et la fonctionnalité de variation dynamique des paramètres par "curseur". La structure itérative de l’algorithme est connue des élèves, mais le traitement à l’intérieur de la boucle est plus complexe que la simple répétition à l’œuvre dans les algorithmes qu'ils ont pu rencontrer (par exemple, le tracé point par point de la courbe d'une

\footnotetext{
${ }^{13}$ La conception et l'utilisation du module du logiciel Casyopée utilisé dans cette tâche sont présentées et discutées par Halbert et al (2019).
} 
fonction). On s’attend donc à ce que les élèves reconnaissent un modèle du câble, mais que la tâche d’interprétation ne soit pas immédiate pour eux.

\begin{tabular}{|c|c|}
\hline $\begin{array}{l}\text { 1. } x \leftarrow-640 \\
\text { 2. // abscisse du point d'ancrage } \\
\text { 3. } y \leftarrow 163 \\
\text { 4. } / / \text { ordonnée du point d'ancrage } \\
\text { 5. } i \leftarrow 1 \\
\text { 6. } V \leftarrow-5+\frac{10}{2 \cdot n} \\
\text { 7. // tension au point d'ancrage }\end{array}$ & $\begin{array}{l}\text { 8. Pour i allant de } 1 \text { à } n \\
\text { 9. } \quad x \leftarrow x+\frac{1280}{n} \\
\text { 10. // abscisse des points de suspension } \\
\text { 11. } \quad y \leftarrow y+\frac{1280}{n} \cdot \frac{V}{H} \\
\text { 12. } / / \text { ordonnée des points de suspension } \\
\text { 13. } \quad V \leftarrow V+\frac{10}{n} \\
\text { 14. // tension dans les segments } \\
\text { 15. Fin Pour }\end{array}$ \\
\hline
\end{tabular}

Fig. 4 : L'algorithme de la tâche C.

La tâche D est dans l'espace de travail des fonctions mathématiques. Les élèves doivent rechercher une fonction $f$ dont la courbe soit un modèle d'un câble porteur (modèle continu). Ils sont informés du fait que la composante horizontale de la tension dans le câble est une constante $H$ et une formule leur est donnée exprimant la composante horizontale de la tension en fonction de l'abscisse dans un repère donné. Ils doivent trouver une formule pour la dérivée de $f$. Puis, en utilisant éventuellement un logiciel mathématique, ils doivent trouver une formule définissant $f$. Le logiciel permettant l'affichage de la représentation graphique de $f$ avec comme arrière plan une image du Golden Gate, les élèves doivent trouver une valeur du paramètre $H$ afin que les deux représentations coïncident. Ils peuvent le faire par ajustement du curseur comme dans la tâche C, ou par calcul. La principale difficulté attendue est dans l’identification de la dérivée de la fonction cherchée comme quotient des composantes verticale et horizontale de la tension. Les élèves devront faire le rapport avec la dérivée en un point comme pente de la tangente à la courbe en ce point, tout en remarquant que la tension est dirigée selon cette tangente.

La table 1 récapitule les modèles, les tâches dans chacun des groupes et les espaces de travail correspondant. 


\begin{tabular}{|l|l|l|}
\hline $\begin{array}{l}\text { Modèle } \\
\text { physique }\end{array}$ & $\begin{array}{l}\text { Tâche A : reconnaître que la } \\
\text { composante horizontale de la } \\
\text { tension dans le câble est } \\
\text { constante et établir une } \\
\text { formule de récurrence pour la } \\
\text { composante verticale. }\end{array}$ & $\begin{array}{l}\text { Espace de travail de la statique } \\
\text { - Sémiotique : suite des tensions. } \\
\text { - Discursive : loi de Newton (système } \\
\text { en équilibre). } \\
\text { - Instrumentale : effectuer des } \\
\text { mesures. }\end{array}$ \\
\hline $\begin{array}{l}\text { Modèle } \\
\text { géométrique } \\
\text { discret (ligne } \\
\text { brisée) }\end{array}$ & $\begin{array}{l}\text { Tâche B : calculer les } \\
\text { coordonnées de points de } \\
\text { suspension, une formule pour } \\
\text { les tensions étant donnée. }\end{array}$ & $\begin{array}{l}\text { Espace de travail de la géométrie } \\
\text { analytique } \\
\text { - Sémiotique : suites définissant les } \\
\text { coordonnées de points. } \\
\text { - Discursive : établir une récurrence. }\end{array}$ \\
\hline $\begin{array}{l}\text { Modèle } \\
\text { algorithmique } \\
\text { (simulation } \\
\text { informatique) }\end{array}$ & $\begin{array}{l}\text { Tâche C : entrer et exécuter } \\
\text { l'algorithme, interpréter le } \\
\text { paramètre } n \text { et ajuster le } \\
\text { paramètre } H \text {. }\end{array}$ & $\begin{array}{l}\text { Espace de travail de l'algorithmique } \\
\text { - Sémiotique : écriture algorithmique. } \\
\text { - Instrumentale : faire fonctionner le } \\
\text { programme et animer des paramètres. } \\
\text { - Discursive : justifier l'algorithme. }\end{array}$ \\
\hline $\begin{array}{l}\text { Modèle continu } \\
\text { (fonction } \\
\text { mathématique) }\end{array}$ & $\begin{array}{l}\text { Tâche D : une formule pour } \\
\text { les tensions étant donnée, } \\
\text { déterminer l'expression de la } \\
\text { dérivée puis celle de la } \\
\text { fonction et calculer le } \\
\text { paramètre } H .\end{array}$ & $\begin{array}{l}\text { D) Espace de travail des fonctions } \\
\text { mathématiques } \\
\text { - Sémiotique : fonctions usuelles. } \\
\text { - Discursive : règles usuelles de calcul. } \\
\text { - Instrumentale : grapher et animer } \\
\text { des paramètres. }\end{array}$ \\
\hline
\end{tabular}

Table 1 : Les modèles, les tâches et les espaces de travail correspondant.

La phase 3 dure aussi 50 min. Les élèves forment les groupes "d’apprentissage".

Chacun de ces groupes rassemble un ou deux élèves de chacun des groupes d'experts de la seconde phase, ayant fait respectivement les tâches A, B, C ou D. Il est demandé à chacun de ces nouveaux groupes de partager les résultats obtenus à la seconde phase et de rédiger un rapport mettant en évidence les points importants. On s'attend à ce que les résultats obtenus dans chacun des groupes soit partiels compte tenu des difficultés soulignées plus haut. Nous faisons l'hypothèse que la compréhension d'un modèle dans un espace de travail donné progresse par mise en relation avec un modèle d'un autre espace. Par exemple, les relations de récurrence dans le modèle géométrique (groupe $B$ ) et le traitement itératif dans le modèle algorithmique (groupe C) devraient s'éclairer l'un l'autre. On attend aussi que les élèves fasse le lien entre ce modèle et l'étude des tensions par le groupe $\mathrm{A}$, et, par passage à la limite, avec le modèle continu (groupe D).

La phase (4) de bilan collectif a pour but de faire le point sur les notions mathématiques et physiques présentes dans ce travail et sur leurs liens, et de mettre en évidence comment les différentes études concourent à la question de la modélisation d’un câble porteur. 


\section{Observation}

Une mise en œuvre a été observée dans une classe de Terminale ( $12^{\mathrm{e}}$ grade) de 35 élèves à la fin du mois de mars. Cette classe était familière avec l'organisation "classe en puzzle". Il s’agissait pour la plupart d'élèves qui réussissent correctement, sans plus. Les contenus en jeu en physique et en mathématiques avaient été abordés dans les cours précédents. Les travaux rendus par les 35 élèves ont été collectés à l’issue de la phase 1. Les phases ultérieures ont été enregistrées. Nous avons mené un entretien complémentaire avec trois élèves choisis par le professeur pour refléter différents profils dans la classe. Nous avons choisi de le faire entre la phase 3 et la phase 4, de façon que les réponses ne soient pas influencées par la synthèse finale.

\section{Phase 1}

Seuls un tiers des élèves ont dessiné un pont suspendu avec des suspentes. Dans les autres dessins, les câbles porteurs semblent soutenir le tablier en son milieu. En réponse à la question (i), ils ont généralement expliqué la non-horizontalité du câble porteur par le poids du tablier du pont, ayant compris à partir de la vidéo que le poids du tablier "plie" le câble. Parmi les exceptions, un des élèves a attribué la forme du câble à son poids comme dans un pont à caténaire. A la question (ii) de savoir si la forme est déterminée par la longueur des suspentes, un tiers des élèves n’a pas donné de réponse, un sur six a répondu oui et la moitié restante a répondu négativement. Seuls deux élèves ont évoqué la tension et la répartition des forces exercées par le tablier. Pour au moins une moitié des élèves, les réponses sont donc compatibles avec une conception du pont avec un tablier rigide soutenu par le câble porteur en son milieu, plutôt que par les suspentes. Deux élèves n’ont pas compris que la question (iii) porte sur une fonction mathématique et ont répondu "alléger le poids du pont". Les autres ont puisé dans le répertoire des fonctions connues. La fonction "carré" ou $x^{2}$, ou parabole est apparue le plus souvent, mais certains ont répondu aussi par "fonction linéaire" et dans quelques cas par "fonction inverse". La fonction carrée étant un modèle adéquat avec l’hypothèse de négligabilité du poids des câbles, dans une perspective "problem solving" ce modèle "phénoménologique" pourrait être quantifié en calculant une équation de la parabole pour un pont donné à l'aide de la longueur du tablier et de la hauteur des piliers. Comme nous l’avons précisé, nous orientons plutôt l’activité des élèves vers des modèles mobilisant des principes physiques et mathématiques et leur compréhension.

Dans le reste de la première phase, après avoir surmonté les difficultés instrumentales avec les montages, les élèves ont correctement enregistré les angles et l'intensité des forces. Ils ont 
reconnu la loi d'équilibre statique, mais ils n'ont généralement pas calculé les composantes. En particulier le fait que la composante horizontale est la même sur les trois dynamomètres dans l'appareil à deux poids n’a pas été relevé. Dans la discussion en classe, avant de répartir les élèves en groupes pour la phase 2, l'enseignant est revenu sur la décomposition d'un vecteur en composantes. En présentant la maquette de la Fig. 3, il a insisté sur l’intensité de la tension et ses variations ("elle augmente quand on se rapproche des piliers et tire très fort"), le rôle des suspentes "qui permet d'avoir un tablier horizontal" et la prédominance du poids du tablier sur celui des câbles et suspentes.

\section{Phase 2}

Nous avons exploité l'enregistrement de quatre groupes, un pour chaque tâche. Les élèves de la tâche A (statique) ont exprimé les relations de récurrence, concluant que la composante horizontale de la tension est constante et que la composante verticale est en progression arithmétique de raison $\Delta P$, mais, comme prévu, ils n’ont pas terminé le calcul de la valeur initiale et n’ont pas abordé la recherche d’une expression explicite de cette composante. Les élèves de la tâche $B$ (géométrie) ont commencé par esquisser un pont avec beaucoup de suspentes, ce qui ne leur a pas permis de considérer des segments modélisant le câble porteur. Ils ont été invités par l'observateur à se limiter à 4 suspentes. Il leur a fallu du temps pour trouver les coordonnées du point d'ancrage et ils ont eu des difficultés à utiliser la formule donnée pour la pente des segments et la distance entre les suspentes afin de calculer les coordonnées du point suivant. Les élèves de la tâche C (algorithmique) ont été très lents à entrer l'algorithme dans l'environnement de programmation. L'affichage de la courbe sur l'écran a été erroné à cause de petites erreurs de recopiage de l'algorithme. Ils n'ont pu corriger que lorsque l'observateur les a aidés à analyser l'algorithme. Ils ont proposé pour le paramètre $n$ la valeur 83 -le nombre de suspentes du pont du Golden Gate- et la représentation est apparue comme une "courbe" plutôt que comme une ligne brisée. N’ayant pas essayé de petites valeurs de $n$, ils ont conclu que ce paramètre n'a pas vraiment d'influence. Ils ont pris en revanche conscience de ce que l'augmentation de la valeur du paramètre $H$ "redresse" le câble. Les élèves de la tâche $\mathrm{D}$ (fonctions mathématiques) ont eu des difficultés à interpréter le fait que la tension est dans la direction de la tangente à la courbe pour obtenir le nombre dérivé en un point.

\section{Phase 3}

Nous avons exploité l'enregistrement d'un groupe réunissant des élèves de chaque groupe d’experts observé en phase 2. Chaque élève a expliqué sa tâche et son travail dans la phase 
précédente. L'enregistrement montre que les autres élèves ont écouté attentivement et demandé des explications supplémentaires. Le paramètre $H$ a été identifié par les élèves comme jouant un rôle dans chaque tâche. Par exemple un élève qui avait fait la tâche $C$ ne s'est plus souvenu de l'effet de l'augmentation de $H$, confondant avec la "hauteur du câble", et a donc proposé pour le compte-rendu "plus $H$ augmente plus le câble descend (par rapport aux points d'ancrage)". Les élèves on fait plusieurs schémas contradictoires et une discussion s'est engagée, l'élève qui avait fait la tâche A insistant sur le fait que $H$ est une tension. Les élèves ont réalisé que "pour $H$ plus grand le câble est plus tendu". Le même élève a aidé à surmonter la difficulté rencontrée par l'élève qui avait fait la tâche D pour trouver la direction de la tangente à la courbe et ensuite la dérivée de la fonction, en déclarant : "il suffit d'intégrer le quotient de $V$ et $H$ (composantes verticales et horizontales de la tension)". En allant plus loin dans la tâche $\mathrm{D}$, les élèves ont été troublés par le paramètre $H$ au dénominateur, certains proposant un $\operatorname{Ln}(H)$ dans la primitive. Ils ont pu calculer cette primitive avec le logiciel mathématique. En revanche, la tâche non terminée B et le lien avec les autres tâches n'ont pas été abordés par ce groupe.

\section{Entretiens d'évaluation et phase 4}

Il s’agissait d'évaluer les liens entre les modélisations dans les trois domaines opérés au cours du travail de groupe. Les élèves ont souligné que la situation était plus complexe que d'habitude ("nous avons dû relier beaucoup de choses différentes") et qu'ils n'étaient pas "habitués à mélanger la physique et les mathématiques". En commentant la première phase ils ont montré comment leur compréhension de la structure d'un pont a progressé : ils ont mentionné le rôle des suspentes et ont fait le lien entre un pont suspendu et un pont en arc relativement à la façon dont le tablier est soutenu. Ils ont également fait le lien entre le montage avec deux poids dans la première phase et un pont suspendu "avec deux suspentes". Ils ont encore eu des difficultés à considérer les pentes des segments dans la tâche B pour trouver les coordonnées des points de suspension. Cependant, ils ont correctement interprété l'algorithme de la tâche $\mathrm{C}$ et ont été capables de relier l'évolution de $x$ et $y$ dans les tâches $\mathrm{B}$ et C. Ils n'ont pas montré clairement que la fonction de la tâche D était la limite de la fonction définie par un algorithme dans la tâche $\mathrm{C}$ : à partir de la visualisation graphique, ils ont pensé que c'était plus ou moins la même fonction pour de grandes valeurs de $n$. La visualisation est alors la façon dont les élèves relient l'algorithme et la fonction mathématique. L'observateur a demandé d'expliquer pourquoi le nombre dérivé en un point de la courbe est le quotient de $V$ et $H$. La réponse attendue était que la tension a la direction de la tangente, mais les élèves ont 
simplement écrit $f^{\prime}(x)=\Delta y / \Delta x=V(x) / H$ sans plus d'explication. La première égalité est commune en physique et la seconde dérive de la définition des composantes dans la tâche $\mathrm{A}$. Ainsi, les élèves ont établi un lien entre l'étude statique et la fonction mathématique sans considérer explicitement le passage à la limite d'un modèle discret à un modèle continu.

L’observation du bilan en classe entière (phase 4) confirme ces données. Le professeur a repris les raisonnements des élèves et les liens entre modèles sans chercher à formaliser le passage à la limite, mais il a souligné l'influence du nombre de suspentes en faisant varier le paramètre $n$ dans le modèle algorithmique de $n=4$ à $n=83$. Il a insisté sur les notions en jeu, sur la décomposition d'une force en composantes et sur le calcul par intégration de la fonction mathématique, dans un souci de "raccrocher au curriculum" à quelques semaines du baccalauréat.

\section{Analyse a posteriori}

La Fig. 5 (à gauche) résume les liens entre les modèles que font les élèves observés. A droite, les liens repérés sont interprétés comme reliant les espaces de travail et spécifiés selon les dimensions en jeu.
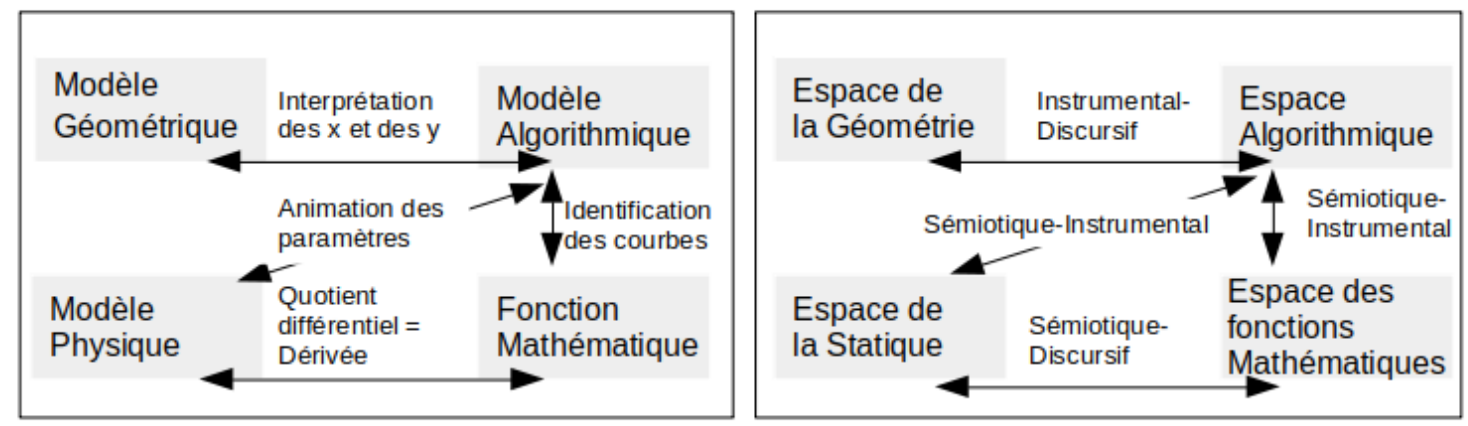

Fig. 5 : Les liens opérés par les élèves à la phase 3, repris de (Lagrange \& Le Feuvre 2019).

Lorsque les élèves interprètent l'évolution des variables $x$ et $y$ dans l'algorithme, en reliantl'initialisation et le corps de la boucle à l'évolution des coordonnées dans le modèle géométrique, cela participe à la validation d'un objet instrumental : le traitement itératif dans l'algorithme donne bien une représentation du câble parce que, comme le dit une élève "c'est ce qu'on a vu tout à l'heure (dans la tâche B), 1280/n c'est la distance qu'on ajoute à $x$, le $\Delta x$, etc." Ce sont les dimensions instrumentale et discursive du travail dans les espaces géométrique et algorithmique qui sont en jeu. Récurrence et itération prennent sens dans cette connexion en référence à l’instrument qui concrétise l’itération.

L'animation du paramètre $H$ dans l'environnement logiciel permet la visualisation de la tension. Après avoir animé le curseur du paramètre $H$, un élève dit : "plus il y a de tension 
plus le câble va être tiré". La représentation du câble par le logiciel a ici une dimension sémiotique (un arc de parabole plus ou moins ouvert) tout comme la tension qui est représentée par un curseur. L’influence de la tension sur la forme du câble est perçue grâce aux représentations sémiotiques "dynamiques" associées à l'instrument qui est ici support d'une simulation.

Nous faisons la même analyse pour le lien entre l'espace algorithmique et les fonctions mathématiques, en ce qui concerne le paramètre $n$. Une élève dit "n c'est le nombre de suspentes, pour $n=3$, ce n'est pas encore une courbe, quand $n$ devient grand, ça devient une courbe" et quand ils font tracer la fonction mathématique, ils reconnaissent le même tracé qu'avec l’algorithme "pour $n$ assez grand".

L'explication que les élèves donnent de la valeur du nombre dérivé de la fonction mathématique s'inspire de la notation des dérivées en physique. Ce sont donc les dimensions discursive et sémiotique qui sont en jeu dans le lien qu'ils opèrent entre l'espace de la statique et celui des fonctions mathématiques. Pour les élèves, le détour par la tangente ne fait pas sens. En revanche, la notation $\Delta y / \Delta x$ renvoie pour les élèves au schéma qui a permis d'exprimer les relations de récurrence sur les tensions dans le modèle discret de la tâche $\mathrm{A}$, et ils transposent ce schéma au cas continu, considérant implicitement la courbe comme une suite de segments infinitésimaux.

Chacun des quatre liens met en lumière des concepts : récurrence et traitement itératif, paramètres dans une fonction, tension, modèles discrets et continus, nombre dérivé. L'analyse dans le cadre des espaces de travail met l'accent sur les dimensions spécifiques dans chaque lien. Kuzniak, Tanguay et Elia (2016, p. 728) décrivent les combinaisons deux à deux de dimensions des espaces de travail comme "des outils précieux pour décrire l'évolution du travail mathématique dans le processus de résolution" ${ }^{14}$. Ils préviennent en particulier que "des activités à fort caractère constructiviste" doivent être conçues pour "éviter d'être emprisonné dans la combinaison instrumental-sémiotique". L’analyse qui précède montre que, dans le travail des élèves, ce type de combinaison existe mais est complété par les deux autres combinaisons incluant la dimension discursive.

\footnotetext{
${ }^{14}$ Notre traduction. Les auteurs nomment "plans verticaux" ces combinaisons, car ils les considèrent comme intervenant dans des "genèses" liant deux plans "horizontaux", respectivement épistémologique et cognitif. Dans cet article, nous partons du postulat selon lequel les connexions vont permettre aux élèves de mieux comprendre les concepts et techniques en jeu. Il faudra aller plus loin sur le terrain du cognitif pour préciser comment cette compréhension se construit et comment elle s’observe, ce pourquoi les "genèses" ont été introduites. Notons que Moutet (2019) définit deux plans épistémologiques, l'un en Physique, l'autre en Mathématiques, et des genèses communes à ces deux plans.
} 
Alors que des liens étaient attendus entre tous les modèles, il n’y a pas de lien entre le modèle géométrique et le modèle physique, ni entre le modèle géométrique et le modèle continu. Les groupes d'experts qui ont eu la tâche B ont été gênés par la complexité des données relatives au pont du Golden Gate et ont peu avancé dans cette tâche. Les capacités des élèves en géométrie analytique et à généraliser un calcul dans le contexte de la tâche ont été surestimées. En revanche, le modèle algorithmique (une simulation dynamique) permet à la fois des liens de type instrumental-sémiotique de par son fonctionnement, mais aussi un lien instrumental-discursif quand il s’agit de justifier le modèle. Notons aussi que les activités de la phase 1 ont permis que la structure du pont et la notion de tension servent de "référent empirique" utile pour les phases ultérieures. On constate que ces éléments "imprègnent" le raisonnement dans chaque espace de travail. Il y a bien intégration du raisonnement dans la réalité avec le raisonnement mathématique. L’obstacle principal est une complexité dans les tâches qui fait que certains liens attendus peuvent ne pas apparaître : les élèves peinent à mettre en œuvre des connaissances et des techniques mathématiques quand le contexte n'est pas familier, montrant ainsi la fragilité de ces connaissances et techniques.

\section{Conclusion}

Nous commençons cette conclusion par un bilan des thèmes abordés. Nous avons montré que dans une approche "problem solving" l'intérêt tend à se porter sur la recherche d'une solution plutôt que sur le ou les modèles et que les approches du type "translation" restent proches d’un schéma de résolution de problème "extra-mathématique" sur deux niveaux séparés, "réel" et "mathématique". Nous avons cherché comment établir une relation plus riche entre contexte du domaine modélisé et mathématiques, sous-tendue par des interactions fortes entre connaissances scientifiques propres à ce domaine et connaissances mathématiques. Une étude de la modélisation en dehors de l'enseignement nous a permis de préciser les situations visées : elles doivent mettre en jeu différents modèles impliquant des connaissances scientifiques diverses et dans lesquels un travail mathématique intervient pour simplifier l'interprétation. Le cadre théorique des "espaces de travail connectés" a permis de concevoir a priori une situation de modélisation et de rendre compte a posteriori du travail des élèves.

Rompant avec une perspective "problem solving" nous situons pour les élèves l'activité comme l'étude d’une question générale, puis nous leur proposons des tâches destinées à explorer des modèles. En caractérisant l'activité sur chaque modèle comme un travail dans un 
espace spécifique, le cadre des espaces connectés aide à organiser et à exploiter un travail de groupe efficace dans un environnement de classe réaliste. Pour les élèves, la modélisation se présente comme l'appropriation active et la mise en relation de modèles de nature variée plutôt qu'une "translation" entre la réalité et les mathématiques. Malgré la complexité de la situation, les élèves observés dans la situation du pont suspendu ont compris les principaux aspects des modèles et les liens entre eux. Ils ont opéré des connexions dans les trois dimensions, sémiotique, instrumentale et discursive du travail dans les différents espaces, qui leur ont permis de progresser dans une compréhension plus globale des concepts en jeu.

En nous écartant des perspectives "problem solving" et "translation", en jetant un pont avec la didactique des sciences et en cherchant des cohérences avec la modélisation en dehors de l'enseignement, nous explorons une nouvelle voie pour la modélisation dans le secondaire. Bien des questions restent ouvertes. Quels modèles retenir ? Le pont suspendu avec quatre modèles conduit à une complexité qui peut être sous-estimée a priori. On peut chercher à diminuer cette complexité, mais aussi considérer qu'elle est inhérente à la modélisation et que même si les élèves n’ont qu'une compréhension partielle, les objectifs de mise en synergie de connaissances et de compréhension du rôle des modèles sont au moins partiellement atteints. Le dispositif de classe en puzzle aide à cela, en faisant travailler deux fois chaque modèle, d'abord dans un espace spécifique, puis en lien avec les autres. Quelle part donner aux élèves dans la construction de chaque modèle et quelles tâches proposer ? Les tâches proposées dans l'exemple sont relativement dirigées pour tenir compte du contexte d'une classe de Terminale. Un travail de recherche vers des tâches plus ouvertes pourrait être nécessaire. Comment rendre compte des apprentissages, au-delà du postulat selon lequel les connexions permettent aux élèves de mieux comprendre les concepts et techniques en jeu ? Il paraît possible d'associer aux "espaces de travail connectés" un cadre "cognitif" comme l’ont fait Psycharis, Kafetzopoulos et Lagrange (à paraître) avec le cadre "Abstraction in Context" ou d'étudier comment la notion de "genèse"15 (Kuzniak, Tanguay \& Elia 2016) pourrait être mobilisée. Comment mettre l'accent sur la composante interprétative des modèles et former ainsi les élèves à une démarche critique ? Le rôle des enseignants de mathématiques n’est pas d'engager les élèves dans des débats sur des choix "sociétaux" mais l’exemple du pont peut faire prendre conscience aux élèves que plusieurs modèles d’une même réalité sont possibles. Un tel travail contribue à réduire le hiatus entre des sciences supposées "positives" et des sciences plus "interprétatives" et à rompre avec une image rigide des sciences "exactes", en considérant la variété de leurs implications dans la vie sociale.

\footnotetext{
${ }^{15}$ Voir note 14.
} 


\section{Remerciements}

Ce travail a été possible grâce à l'accueil très favorable du lycée Cassin (Montfort su Meu, France) ainsi que grâce au soutien de l'Institut de Recherche sur l'Enseignement des Mathématiques de Rennes. Remercions aussi les relecteurs pour la précision de leurs très nombreuses remarques et leur bienveillance.

\section{Références}

Blanquet E., et Picholle P. (2015). Premiers contacts avec la modélisation scientifique à l'école. Dans T. Amory et B. Evrard (Éds.), Les Modèles, des incontournables pour enseigner les sciences! (pp. 45-55) Louvain-la-Neuve : de Boeck.

Blum, W., et Ferri, R. B. (2009). Mathematical modelling: Can it be taught and learnt? Journal of Mathematical Modelling and Application, 1(1), 45-58.

Bouleau, N. (2014). La modélisation critique. Versailles: Quae.

Czocher, J. A. (2018). How does validating activity contribute to the modeling process? Educational Studies in Mathematics, 99(2), 137-159.

Derouet, C., et Parzysz, B. (2016). How can histograms be useful for introducing continuous probability distributions? ZDM - The International Journal on Mathematics Education, 48(6), 757-773.

Raymond Duval (1995). Semiosis et pensée humaine. Bernes : Peter Lang.

Halbert, R., Lagrange, J.B., Le Bihan, C., Le Feuvre, B., et Manens, M.C. (2019). Aider à la compréhension des fonctions avec un logiciel : les fonctions définies par un algorithme, Repères IREM, 117, 35-54.

Kaiser, G., et Schwarz, B. (2010). Authentic modelling problems in mathematics education. Examples and experiences. Journal for Didactics of Mathematics, 31(1), 51-76.

Kuzniak, A. (2013). Teaching and learning geometry and beyond. Dans B. Ubuz et al. (Eds.) Proceedings of CERME 8, Antalya, Turkey.

Kuzniak, A., Tanguay, D., et Elia, I. (2016). Mathematical Working Spaces in schooling: an introduction. ZDM-Mathematics Education, 48(6), 721-737.

Lagrange, J.B., et Le Feuvre, B. (2019). Espaces de travail mathématique connectés au lycée : un pont entre les mathématiques et les sciences physiques. Modélisation des câbles d'un pont suspendu par une fonction numérique spécifique. Dans M. Abboud (Éd.), Actes du colloque EMF 2018 (pp.1205-1213). Paris : IREM de Paris. 
MEN/DGESCO-IGEN (2013). Les compétences mathématiques au lycée. Retrieved from https://cache.media.eduscol.education.fr/file/Mathematiques/90/0/Competences mathemati ques Lycee 282900.pdf

Minh T. K., et Lagrange J.B. (2016). Connected functional working spaces: a framework for the teaching and learning of functions at upper secondary level. ZDM Mathematics Education, 48, 793-807 (2016). https://doi.org/10.1007/s11858-016-0774-z.

Moutet, L. (2019). The extended theoretical framework of Mathematical Working Space (extended MWS): potentialities in physics. Dans U. T. Jankvist, M. Van den HeuvelPanhuizen et M. Veldhuis (Eds.) Proceedings of the Eleventh Congress of the European Society for Research in Mathematics Education (pp. 1240-1247). Utrecht University and ERME.

Psycharis, G., Kafetzopoulos, G., et Lagrange J.B. (à paraître). A framework for analysing students' learning of function at upper secondary level: Connected Working Spaces and Abstraction in Context. Dans A. Clark-Wilson (Éd.) Mathematics Education in the Digital Age: Learning, Practice and Theory. Springer

Roux S. (2011). Pour une étude des formes de la mathématisation. Dans H. Chabot et S. Roux (Éds.), La mathématisation comme problème (pp.3-38). Editions des archives contemporaines.

Sanchez, E. (2008). Quelles relations entre modélisation et investigation scientifique dans l'enseignement des sciences de la terre ? Éducation et Didactique 2 (2), 97-122.

Sierpinska, A. (1999). Interaction des perspectives épistémologique, cognitive et didactique. Dans G. Lemoyne, F. Conne (Éds.), Le cognitif en didactique des mathématiques (pp. 151-176). Montréal : Presses de l’Université de Montréal. DOI : $10.4000 /$ books.pum.14684.

Varenne, F. (2014). Chapitre 1. Épistémologie des modèles et des simulations : tour d'horizon et tendances. Dans J-M. Lévy (Éd.), Les modèles, possibilités et limites: Jusqu’où va le réel ? (pp. 13-46). Paris: Editions Matériologiques. 


\section{Annexe : quatre modèles d'un câble porteur dans un pont suspendu}

\section{Un modèle statique discret}

Le modèle statique discret dérive du nombre fini de suspentes : un câble porteur est représenté comme un ensemble de $n$ barres rigides, en commençant et en terminant au niveau des points d'ancrage (partie supérieure d'un pilier), et séparés par ( $n-1)$ points de suspension reliant le câble et les suspentes. On considère que chacune des (n-1) suspentes supporte une portion de longueur $L / n$ du tablier, deux portions de longueur $L /(2 n)$ étant supportées par chacun des piliers. La modélisation de la tension dans l'un des câbles porteurs peut être effectuée en considérant la suite des tensions sur chaque segment et pour chacune des tensions, les valeurs de la composante horizontale et de la composante verticale $\left(H_{i} ; V_{i}\right)$. La loi d'équilibre statique, appliquée à chaque point de suspension, implique que la composante horizontale est la même dans toutes les barres : $H_{i}=H$ pour tous les points $i$. Cela implique aussi que la suite des valeurs de la composante verticale est en progression arithmétique de raison la valeur $\Delta P$ du poids d'une partie du tablier supporté par une suspente. Comme chacun des deux câbles principaux supporte la moitié du tablier, on a $\Delta P=P /(2 n), P$ étant le poids du tablier. $V_{\mathrm{n}}=V_{0}+(n-1) P /(2 n)$. Par symétrie $V_{n}=-V_{0}$ d'où la relation de récurrence :

$$
V_{i+1}=V_{i}+\frac{P}{2 n} \text { et } V_{0}=\frac{-P}{4}+\frac{P}{4 n}
$$

\section{Un modèle géométrique discret}

Connaissant la position d'un point d'ancrage il est possible de calculer la suite des coordonnées des points de l'ensemble des segments modélisant le câble. $\mathrm{M}_{0}$ et $\mathrm{M}_{n}$ étant les points d'ancrage sur les piliers et $\mathrm{M}_{1}, \mathrm{M}_{2}, \ldots \mathrm{M}_{n-1}$, les points où les suspentes sont fixées au câble. La suite des abscisses des $M_{i}$ est en progression arithmétique de raison $L / n$. La pente d'un segment $\left[\mathrm{M}_{\mathrm{i}}, \mathrm{M}_{\mathrm{i+1}}\right]$ est le rapport entre les composantes verticale et horizontale de la tension dans ce segment, d'où la formule de récurrence pour les ordonnées des $\mathrm{M}_{\mathrm{i}}$ :

$y_{i+1}=y_{i}+\frac{L}{n} \frac{V_{i}}{H}$. Avec la formule de récurrence donnée par le modèle statique pour $V_{\mathrm{i}}$, il est possible de calculer les $y_{i}$.

\section{Un modèle algorithmique discret}

Un algorithme (Fig. 5) permet de définir une fonction affine par morceaux en systématisant la construction géométrique. Les données proviennent du Pont du Golden Gate (tablier de 
longueur $1280 \mathrm{~m}$ et de poids 20 méga newtons, hauteur des piliers de 163 mètres) et l'origine du système de coordonnées est au milieu du pont.

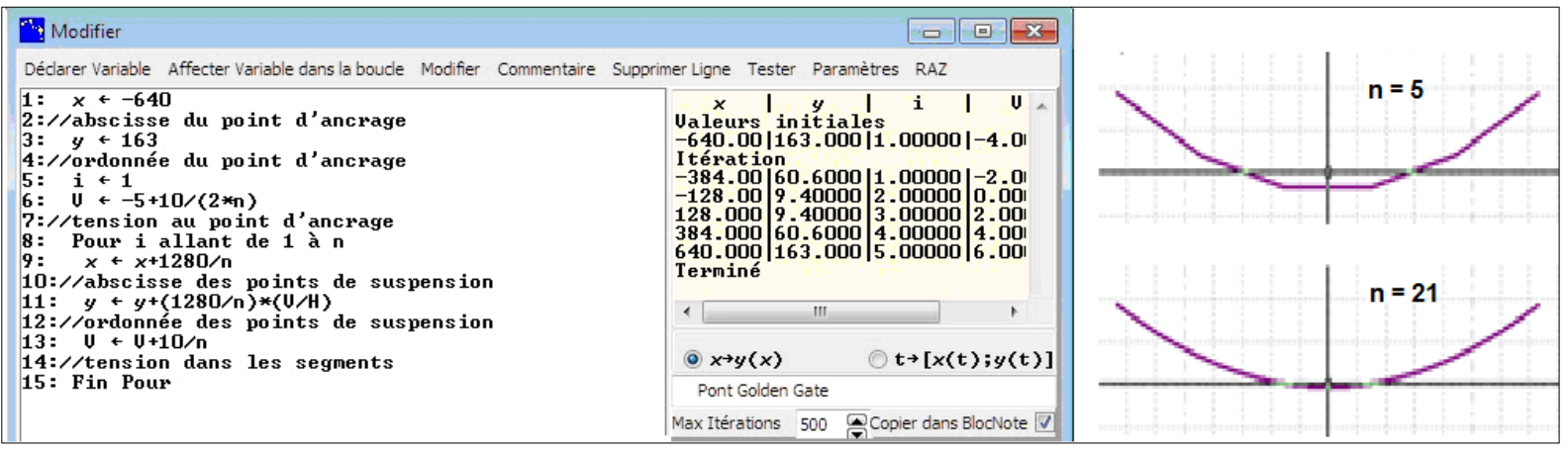

\section{Fig. 6 : L'algorithme et la visualisation}

A ce stade, $H$ est inconnu et donc paramétrique, et nous choisissons également un paramètre $n$ pour le nombre de suspentes afin d'obtenir des représentations pour plusieurs valeurs de $n$. En utilisant un environnement de programmation, nous obtenons des représentations pour des valeurs variées de $H$ et $n$. Pour les petites valeurs de $n$, la représentation est un ensemble de segments. Pour des valeurs plus grandes, elle paraît une courbe. On peut ajuster $H$ afin que le câble atteigne une hauteur donnée au-dessus du pont. Plus cette composante horizontale est grande, plus le câble s'élève au-dessus du tablier.

\section{Un modèle continu}

Étant donné le grand nombre de suspentes, on peut chercher une courbe, limite de l'ensemble des segments modélisant le câble lorsque ce nombre tend vers l'infini, et que donc la distance entre suspentes tend vers zéro. Avec un choix de l'origine du repère au milieu du tablier, on considère alors le câble principal comme modélisé par la courbe d'une fonction $f$ définie sur

[-L/2; $L / 2]$. La tension en un point du câble d'abscisse x est la limite de la tension sur une suite de segments d'amplitude tendant vers zéro et encadrant $\mathrm{x}$ dans le modèle discret. Comme la composante horizontale de la tension dans le modèle discret est une constante que nous avons notée $H$, la composante horizontale de la tension dans le modèle continu est cette même constante. Par un passage à la limite depuis le modèle en statique, on obtient la composante verticale de la tension : . $V(x)=x \cdot \frac{P}{2 L}$ La tension étant dirigée selon la tangente à la courbe, le nombre dérivé de la fonction cherchée en un point $x$ est $: \frac{V(x)}{H}$. Avec les données du pont 
du Golden Gate, nous avons $f^{\prime}(x)=\frac{x}{128 H}$ puis par intégration $f(x)=\frac{x^{2}}{256 H}+C$. La courbe est un arc de parabole. En prenant $C=0$ (câble passant au ras du tablier), l'équation $f(640)=163$ donne $H$ peu différent de 9,8. 